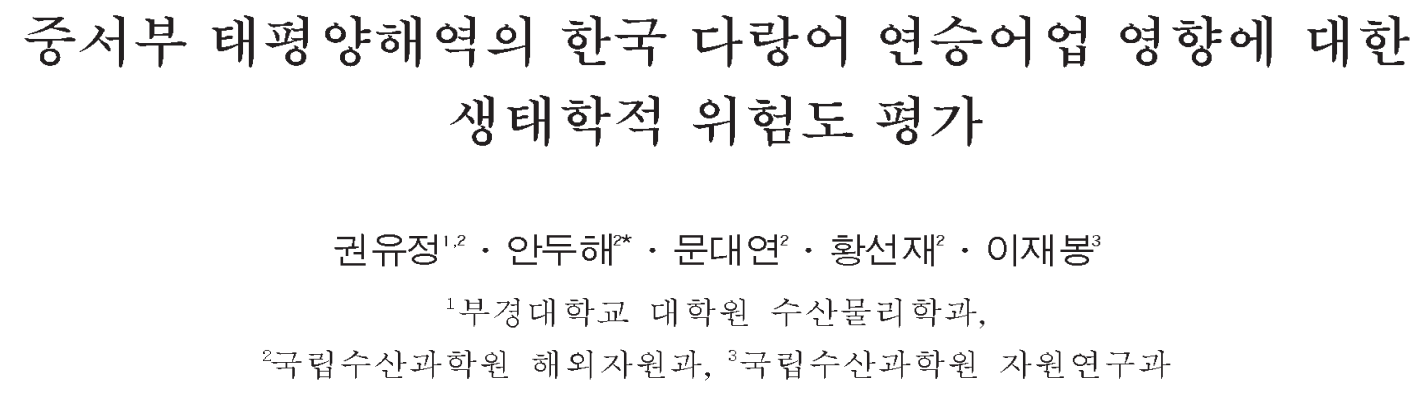

\title{
An ecological risk assessment for the effect of the Korean tuna longline fishery in the Western and Central Pacific Ocean
}

\author{
Youjung Kwon ${ }^{1,2}$, Doo-Hae $\mathrm{AN}^{2 *}$, Dae Yeon $\mathrm{MooN}^{2}$, \\ Seon Jae HwANG ${ }^{2}$ and Jae Bong LEE $^{3}$ \\ ${ }^{1}$ Department of Fisheries Physics, Graduate School, Pukyong National University, Busan, 608-737, Korea \\ ${ }^{2}$ Distant Water Fisheries Resources Division, National Fisheries Research and Development Institute, \\ Busan, 619-902, Korea \\ ${ }^{3}$ Fisheries Resources Research Division, National Fisheries Research and Development Institute, \\ Busan, 619-902, Korea
}

Ecological risk assessment(ERA), developed in Australia, can be used to estimate the risk of target, bycatch and protected species from the effects of fishing using limited data for stock assessment. In this study, we employed the ERA approach to estimate risks to tunas, billfishes, sharks, sea turtles and other species by the Korean tuna longline fishery in the Western and Central Pacific Ocean using productivity and susceptibility analyses of the ERA based on low( $<1.30)$, medium(1.30-1.84) and high risk( $>1.84)$. Albacore, bigeye, yellowfin, skipjack and bluefin tunas were generally evaluated in the medium risk. The susceptibility of tuna species, however, had higher risks than the productivity. Billfishes were also at medium risk, while sharks were at high risk by the tuna longline fishery. The risk of productivity was generally high, because most sharks caught by the tuna longline fishery have high longevities, i.e., over 10 years, including ovoviviparous species. Susceptibility, which is related with the selection of fishing gear, was also high, because the longline fishery has no gear modifications to prevent bycatch of protected species. Not only target tuna species were influenced by the tuna longline fishery in the Western and Central Pacific Ocean, but also nontarget species, such as pomfret, mackerels rays, sea turtle were done. Ecosystem-based fishery assessment

*Corresponding author: dhan@nfrdi.go.kr, Tel: 82-51-720-2320, Fax: 82-51-720-2337 
tools, such as productivity and susceptibility analysis(PSA), have the ability to provide broad scientific advice to the policy makers and stakeholders.

Key words : Ecological risk assessment, Bycatch, Longline fishery, Pacific Ocean

\section{서 론}

전 세계 대부분의 상업적 규모의 어업에서는 상업적으로 이용가치가 없거나 크지 않은 부수 어획종들은 대부분이 폐기되거나 일부만 이용 되고 있어 정 확한 어획실 태에 대한 통계 및 조사 가 실시되고 있지 않다. 그로인한자료의 부족으 로 자원상태에 대한 평 가가 거의 이루어지지 않고 있으며 우리가 인지하지 못하는 상태에서 고 갈되는 심각한 경 우가 빈 번하게 발생하고 있다. 따라서 현재 어업에서 부수어획에 관한 문제는 전 세계적으로 중요한 문제로 부각되고 있다 (Gilman et al., 2008; Yokota et al., 2006).

$\mathrm{FAO}$ 는 대 상생태계의 종 다양성(biodiversity) 및 지속가능한 어업을 유지하기 위해서는 상업 어업의 주목표종에 대한 정 량적인 자원평 가 뿐 만 아니라 비목표종에 대해서 도 생태계의 관점 에서의 예 방적 접 근법(precautionary approach)이 도입되어야 한다고 주장하고 있 다(FAO, 2007a). 오늘날 전 세 계적으로 국가단위 혹은 지역수산 관리기구를 중심으로 해 당해역의 상업적 중요 종에 대해서는 정 량적 평 가를 실시하지만, 현 실 적으로 대부분의 해역에는 비상업 적 어종 즉, 부 수 어획종들에 대해서는 거의 평 가가 이루어지 지 않고 있다. 최근에 이러한 부수 어획종들에 식별 및 평가관리를 위해 포괄적인 생태계 접근 법을 통한 생태계기반 어업관리(ecosystembased fisheries management, EBFM) 기법이 제시 되었다(Kirby, 2006). 생태계기반 어업관리는 생 태계의 붕괴 예방, 어쩔 수 없는 환경, 어업 등의 변화에 대한 위험의 최 소화, 어업으로 인 한 장기 간 사회 - 경제 적 이윤획 득 및 예 방적 접 근법 에 그 목적이 있다(Pikkitch et al., 2004). 생 태계기반
어업관리의 한 방법으로 제시된 생태학적 위험 도평 가(ecological risk assessment, ERA)는 어업 의 영향을 받는 대상생태계의 모든 종을 대상으로 위험도평가를 하여, 자원의 재생산율(reproductive rate)이 낮은 높은 단계 의 위험도로 평가 된 종을 파악한 후 향후 분석 또는 관리를 위해 고려된 방법이다(Kirby and Moloney, 2007). 생 태 학적 위험도평가는 현재 호주에서 관리전략평 가(management strategy evaluation)의 하나로 상 어 어업 의 자원관리에 사용되 고 있으며(Smith et al., 2007), 중서부태평 양수산위 원회(Western and Central Pacific Fisheries Commission, WCPFC)에 서는 2006 년 부터 부수 어획종들의 향후 자원평 가 및 관리를 위한 예 방적 접 근법으로 개 발되 고 있다(Kirby, 2006).

지난 20년 동안 중서부태평양해역의 한국다 랑어연 승어업 의 주목표종은 눈 다랑어(Thunnus obesus)를 포함한 다랑어류가 전체 어획량의 약 $90 \%$ 였으며, 그 외 부수어획종이 $10 \%$ 의 비율로 어획되 었다(Moon et al., 2007). 본 연 구에서는 중 서부태평양해역 의 한국다랑어 연승어업 에 의해 영항을 받는 목표종 뿐만 아니라 부수 어획되는 모든 종에 대한 생태학적 위험도평가를 실시하 여, 향후 자원관리 및 평 가를 위한 예방적 접근 법으로 사용하고자 한다.

\section{재료 및 방법}

본 연 구에 는 Fig. 1 에서와 같이 2005년 7 월 15 일 부터 2008 년 2 월 29 일 까지 중서부태평양해 역 한국 다랑어 연 승어업을 대상으로 실시한 충 7 회의 과학옵서버 승선조사 시 총 355 번의 투. 양승 자로가 사용되 었다. 


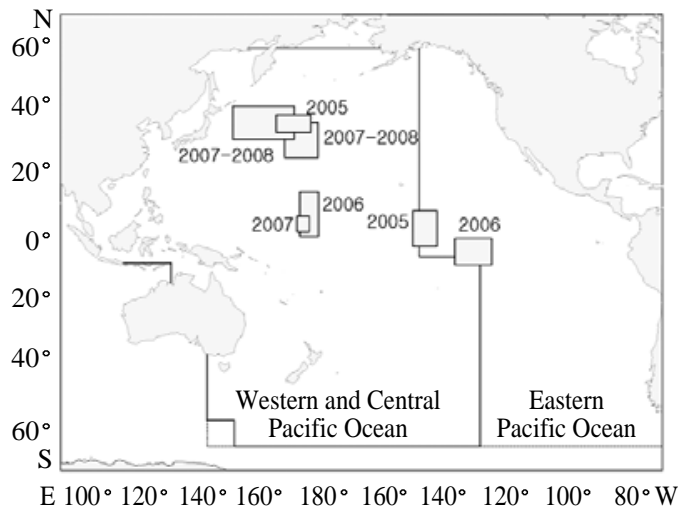

Fig. 1. Survey areas of the Korean tuna longline fisheries by the Korean scientific observers during 2005-2008 in the Western and Central Pacific Ocean.

본 연구에서 이융한 생태학적 위험도 평가 (ecological risk assessment, ERA) 방법은 모델을 기반으로 하는 정량적 자원평가(quantitative stock assessment)에 필요한 어 업자료 및 생물학 적 정보 등이 부족할 때 사웅하는 준정량적 분석 법(semi-quantitative analysis) 중의 하나인 생산 력 - 민감도 분석법(productivity and susceptibility analysis, PSA)을 사용하였 다(Stobutzki et al., 2001, Smith et al., 2007). PSA 는 정 량적 자원평 가 에서 사용되는 자원량 추정식 식(1)에 의해 설명 될 수 있다.

$$
\frac{d B}{d t}=r B\left(1-\frac{B}{K}\right)-q E B
$$

여 기서 $B$ 는 자원량 혹은 자원 개체수, $r$ 은 내적 증가량, $K$ 는 환경 수융력, $q$ 는 어획률, $E$ 는 어획 노력이다. PSA 에서 생산력 은 어획활동으로 받 은 영향의 회복율을 나타내므로 식(1)에서 내 적 증가량 $(r)$ 으로 표현 될 수 있으며, 민감도는 어 획활동으로 대 상어 종의 영항을 받은 정 도를 나 타내므로 식(2)에서는 어획률 $(q)$ 로 표현 될 수 있다.

한국 다랑어 연승어입 의 영향에 대한 ERA 평 가를 위해 과학옵서버 승선조사 시 어 획된 총 47 종을 Table 1 과 같이 다랑어류, 새치류, 상어류,
바다거북류 및 기타어종 등 크게 5 개의 군으로 나누었다. 다랑어류에는 눈다랑어(Thunnus obesus), 날개다랑어(Thunnus alalunga) 둥 5종, 새치류에는 황새치(Xiphias gladius), 녹새치 (Makaira mazara) 등 6종, 상어류에는 12 종, 바다 거북류에는 3 종, 기타 어종에 는 21 종이 각각 포 함되었다.

각 어종의 ERA 평 가는 Table 2 에 제 시된 생 산 력 과 민감도에 대한 지표들(indicators) 과 이들 지포별 기준점 들(reference points) 에 의해 평 가 되었다(Stobutzki et al., 2001). PSA 는 어 구에 의 해 영항을 받은 어종들의 생물학적 회복율에 초 점을 두고 있기 때문에 객관적인 생물학적 자료 가 필요하므로, 생산력 평 가를 위해 사용된 생물 학적 자료는 FishBase(http:// www.fishbase.net) 를 이용하였다. 생산력 평 가를 위한 지표로는 최대 수명 및 체장, 성숙연령 및 체장 등의 자료가 사 용되 었으며, 민감도 평가에 사용된 지표로는 어 구와 어종이 마주치는 정도(encounterability), 어 종의 분포, 어획 또는 포획 후 사망 정도 등이다. 특히, Fig. 2 와 같이 어구와 어종이 마주치는 정 도에 대한 기준점은 총 7회의 승선조사 시에 사 용된 연승어구들의 투승 시 최대수심에서 부표 줄까지의 평균값을 사용하였다.

각 어종의 분포수심에 따라이 지표에 대한 위 험도가 평 가되는데, 분포수심이 어 구의 최대전 개수심인 309m이내에 서식하는 종은 연 승어구 와 마주칠 확률을 $100 \%$ 로 가정하여 높은 위험 도(2)로 평 가되며, 반대로 분포수심이 $309 \mathrm{~m}$ 이 하 인 어종에 대해서는 어구와 마주칠 확률을 $0 \%$ 로 가정하여 낮은 위험도(0)로 평 가되 었다. 연 승 어 구는 트롤어구나 자망어구 등과 같이 어종의 체 장에 따른 선택성을 가지고 있지 않기 때문에 본 연 구에서는 모든 어종에 대한 선 택성 은 높은 위험도로 가정하였다.

평 가를 위한 생물학적 자로가 없을 때는 같은 과(family)에 속하는 유사종끼리 묶어 자료를 사 용하였으며, 유사종에 대한 자료까지 없을 경우 
Table 1. Species composition of species caught by the Korean tuna longline fisheries during 2005-2008 in the Western and Central Pacific Ocean

\begin{tabular}{|c|c|c|}
\hline Group & English name & Scientific name \\
\hline \multirow{5}{*}{$\begin{array}{c}\text { Tunas } \\
\text { (5) }\end{array}$} & Bigeye tuna & Thunnus obesus \\
\hline & Albacore & Thunnus alalunga \\
\hline & Yellowfin tuna & Thunnus albacares \\
\hline & Skipjack tuna & Katsuwonus pelamis \\
\hline & Bluefin tuna & Thunnus thynnus \\
\hline \multirow{6}{*}{$\begin{array}{l}\text { Billfishes } \\
\text { (6) }\end{array}$} & Swordfish & Xiphias gladius \\
\hline & Blue marlin & Makaira mazara \\
\hline & Striped marlin & Tetrapturus audax \\
\hline & Indo-Pacific sailfish & Istiophorus platypterus \\
\hline & Shortbill spearfish & Tetrapturus angustirostris \\
\hline & Black marlin & Makaira indica \\
\hline \multirow{12}{*}{$\begin{array}{c}\text { Sharks } \\
(12)\end{array}$} & Blue shark & Prionace glauca \\
\hline & Mako shark & Isurus oxyrinchus \\
\hline & Bigeye thresher shark & Alopias superciliosus \\
\hline & Oceanic whitetip shark & Carcharhinus longimanus \\
\hline & Salmon shark & Lamna ditropis \\
\hline & Smooth hammerhead shark & Sphyrna zygaena \\
\hline & Scalloped hammerhead shark & Sphyrna lewini \\
\hline & Crocodile shark & Pseudocarcharias kamoharai \\
\hline & Silky shark & Carcharhinus falciformis \\
\hline & Galapagos shark & Carcharhinus galapagensis \\
\hline & Grey reef shark & Carcharhinus amblyrhynchos \\
\hline & Dogfish shark & Isistius brasiliensis \\
\hline \multirow{3}{*}{$\begin{array}{c}\text { Turtles } \\
\text { (3) }\end{array}$} & Green sea turtle & Chelonia mydas \\
\hline & Loggerhead sea turtle & Caretta caretta \\
\hline & Olive ridley sea turtle & Lepidochelys olivacea \\
\hline \multirow{21}{*}{$\begin{array}{c}\text { Other } \\
\text { species } \\
(21)\end{array}$} & Escolar & Lepidocybium flavobrunneum \\
\hline & Opah & Lampris guttatus \\
\hline & Sickle pomfret & Taractichthys steindachneri \\
\hline & Oilfish & Ruvettus pretiosus \\
\hline & Wahoo & Acanthocybium solandri \\
\hline & Lancetfish & Alepisaurus ferox \\
\hline & Shortnose Lancetfish & Alepisaurus brevirostris \\
\hline & Dolphinfish & Coryphaena hippurus \\
\hline & Sharptail mola & Masturus lanceolatus \\
\hline & Pelagic stingray & Dasyatis violacea \\
\hline & Crested oarfish & Lophotus lacepede \\
\hline & Snake mackerel & Gempylus serpens \\
\hline & Black pomfret & Taractes rubescens \\
\hline & Manta ray & Mobula japanica \\
\hline & Ocean sunfish & Mola mola \\
\hline & Great barracuda & Sphyraena barracuda \\
\hline & Razorback scabbardfish & Assurger anzac \\
\hline & Slender sunfish & Ranzania laevis \\
\hline & Rainbow runner & Elagatis bipinnulata \\
\hline & Suck fish & Remora remora \\
\hline & Flyingfish & Prognichthys gibbifrons \\
\hline
\end{tabular}


Table 2. Attributes, indicators and reference points for ecological risk assessment

\begin{tabular}{|c|c|c|c|c|}
\hline \multirow{2}{*}{ Attributes } & \multirow{2}{*}{ Indicators } & \multicolumn{3}{|c|}{ Reference points } \\
\hline & & $\operatorname{Low}(0)$ & Medium(1) & $\operatorname{High}(2)$ \\
\hline \multirow{6}{*}{ Productivity } & Maximum age(year) & $<10$ & $10-25$ & $25<$ \\
\hline & Age at maturity(year) & $<5$ & $5-10$ & $10<$ \\
\hline & Size at maturity(cm) & $<40$ & $40-200$ & $200<$ \\
\hline & Maximum size(cm) & $<100$ & $100-300$ & $300<$ \\
\hline & Reproductive strategy & Broadcast spawners & Demersal spawners & Live bearer \\
\hline & Trophic level & $<2.75$ & $2.75-3.25$ & $3.25<$ \\
\hline \multirow{5}{*}{ Susceptibility } & Encounterability(m) & $309<$ & $167<$ & $25-309$ \\
\hline & Global distribution & Worldwide & Hemisphere & Locality \\
\hline & Adult habitat overlap with juvenile & Low rate & Medium rate & High rate \\
\hline & Selectivity & \multicolumn{3}{|c|}{ No selectivity for longline fishery } \\
\hline & Post-capture mortality & Alive & Barely alive & Dead \\
\hline
\end{tabular}

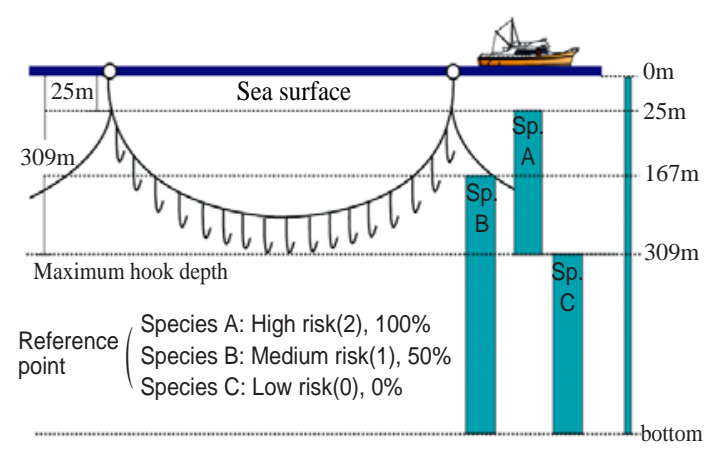

Fig. 2. An example of indicators for ecological risk assessment. This example shows the overlap with fishing effort by is the Korean tuna longline fisheries during 2005-2008 in the Western and Central Pacific Ocean.
에 위험의 정도를 판단 할 수 없어 모두 높은 위 험 도로 가정하였다.

Fig. 3은 각 어종의 위험도를 지표별, 특성 (attribute) 별로 위험도를 도식화하였 다. Fig. 3(a) 는 지표별 위험도는 각 어종의 성숙체장대비 어 획 된 어종의 평균체 장에 대한 지 표를 비교하여 위험도를 평가하였다. 각 어종의 성숙체장은 FishBase자료를 근거로 하였으며, 어획종별 평 균체 장은 2005 -2008년까지 어획된 어종에서 과학옵서버에 의해 측정된 각 어 종들의 산술평 균한 값을 사용하였다. Fig. 3(b)에서 특성별 위 험 도는 각 어종의 지 표들의 위험도를 산술평균 하여 평 가하였으며, 낮은 위험도(<1.30), 중간

(b)

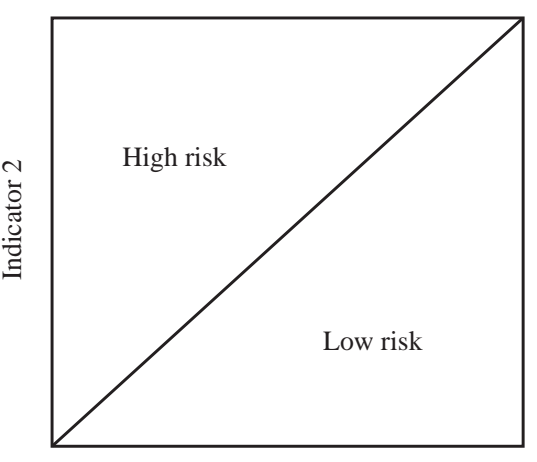

Indicator 1

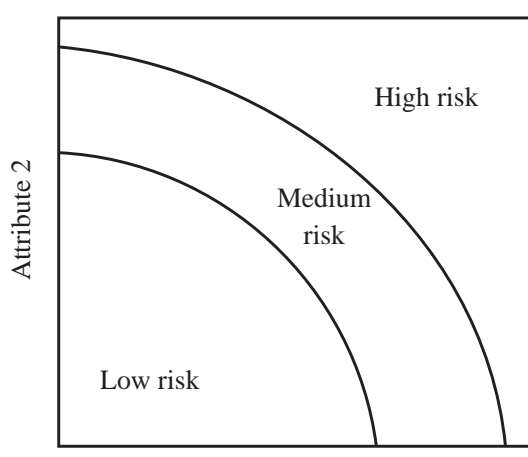

Attribute 1

Fig. 3. Plots between (a) indicators and (b) attributes. Indicators are length at maturity and captured mean length of species, and Attributes are productivity and susceptibility of species caught by the Korean tuna longline fisheries during 2005-2008 in the Western and Central Pacific Ocean. 
위험도(1.30 - 1.84), 놀은 위험도(1.84〈)로 도식 화하였다.

각 어종에 대한 특성별 위험도 크기는 식(2)에 의해 계산되었다.

$$
D_{i}=\sqrt{P_{i}^{2}+S_{i}^{2}}
$$

여기서, $D_{i}$ 는 종의 위험도의 크기, $P_{i}$ 는 $i$ 종의 생 산력에 대한 위험도, $S_{i}$ 는 $i$ 종의 민감도에 대한 위험도이다. 낮은 위험도 구간은 높은 재 생산율
과 어구의 낮은 영향력, 반대로 높은 위험도 구 간에서 는 낮은 재생산율과 어 구의 놀은 영향력 을 의미한다.

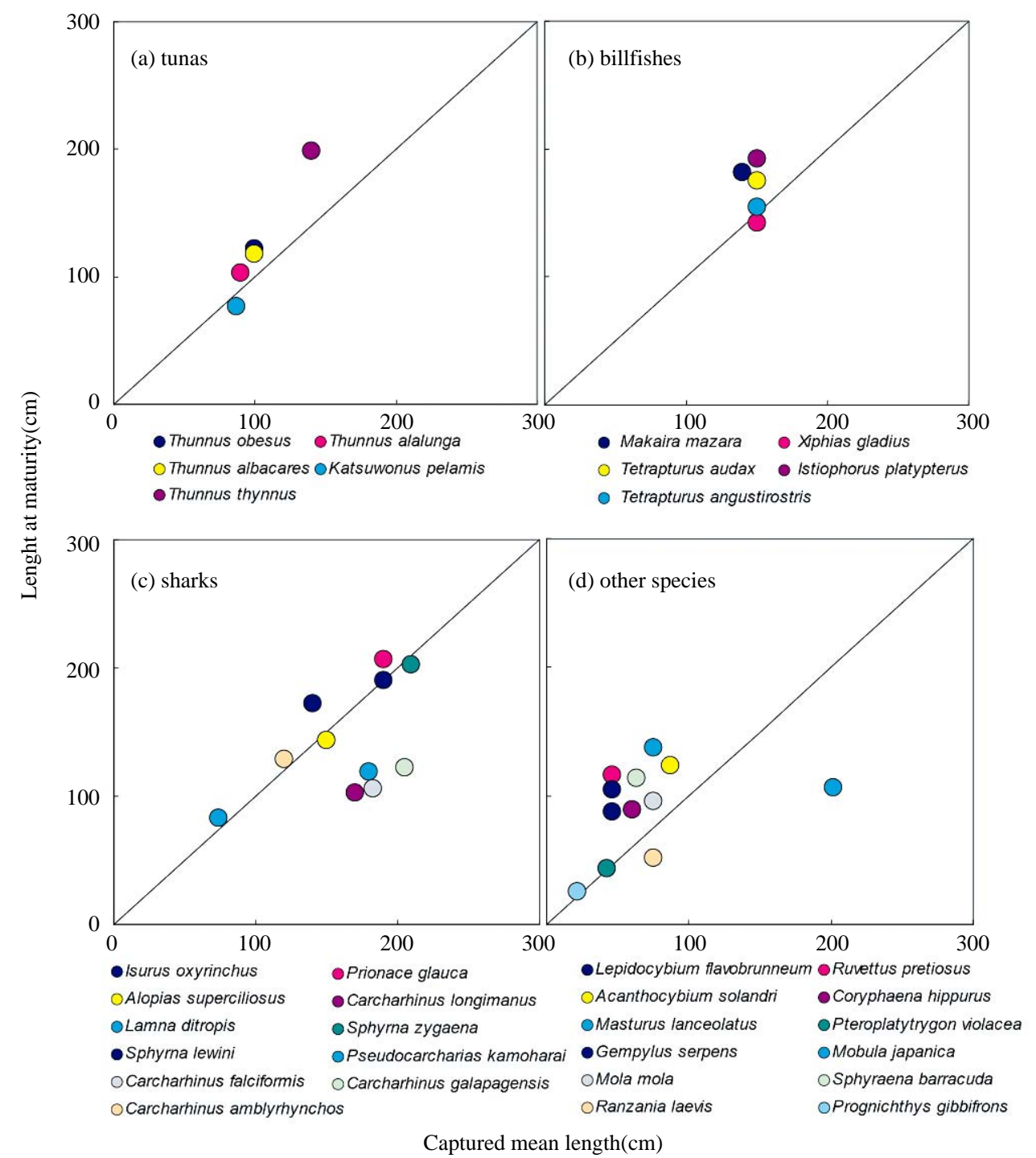

Fig. 4. Plot between indicators of species caught by the Korean tuna longline fisheries during 2005-2008 in the Western and Central Pacific Ocean. 
평균체장이 작은 낮은 위험 도를 나타냈다. 그러 나 바다거북류에 대한 체장자료가 없어 바다거

북류의 지표별 위험도는 평 가되지 못했다. Fig. 4(a)에서 대부분의 다랑어류는 높은 위험도를 보 였으며, 그 중 참다랑어(Thunnus thynnus) 가 가장 높은 위험도를 나타낸 반면, 가다랑어(Katsu-

Table 3. Risk score of productivity and susceptibility and distance of species caught by the Korean tuna longline fisheries during 2005-2008 in the Western and Central Pacific Ocean

\begin{tabular}{|c|c|c|c|c|}
\hline Group & Species & Productivity risk score & Susceptibility risk score & Distance \\
\hline \multirow{5}{*}{ Tunas } & Thunnus obesus & 0.857 & 1.000 & 1.317 \\
\hline & Thunnus alalunga & 0.714 & 1.000 & 1.229 \\
\hline & Thunnus albacares & 0.714 & 1.000 & 1.229 \\
\hline & Katsuwonus pelamis & 0.714 & 0.750 & 1.036 \\
\hline & Thunnus thynnus & 1.143 & 1.250 & 1.694 \\
\hline \multirow{6}{*}{ Billfishes } & Xiphias gladius & 1.167 & 1.000 & 1.537 \\
\hline & Makaira mazara & 1.286 & 1.250 & 1.793 \\
\hline & Tetrapturus audax & 1.000 & 1.250 & 1.601 \\
\hline & Istiophorus platypterus & 1.000 & 1.250 & 1.601 \\
\hline & Tetrapturus angustirostris & 1.000 & 1.000 & 1.414 \\
\hline & Makaira indica & 1.143 & 1.000 & 1.519 \\
\hline \multirow{12}{*}{ Sharks } & Prionace glauca & 1.714 & 1.250 & 2.122 \\
\hline & Isurus oxyrinchus & 2.000 & 1.750 & 2.658 \\
\hline & Alopias superciliosus & 1.857 & 1.250 & 2.239 \\
\hline & Carcharhinus longimanus & 1.714 & 1.750 & 2.450 \\
\hline & Lamna ditropis & 2.000 & 1.250 & 2.358 \\
\hline & Sphyrna zygaena & 2.000 & 1.500 & 2.500 \\
\hline & Sphyrna lewini & 1.857 & 1.750 & 2.552 \\
\hline & Pseudocarcharias kamoharai & 1.667 & 1.250 & 2.083 \\
\hline & Carcharhinus falciformis & 1.857 & 1.500 & 2.387 \\
\hline & Carcharhinus galapagensis & 1.857 & 2.000 & 2.729 \\
\hline & Carcharhinus amblyrhynchos & 1.714 & 1.500 & 2.278 \\
\hline & Isistius brasiliensis & 2.000 & 1.000 & 2.236 \\
\hline \multirow{3}{*}{ Turtles } & Chelonia mydas & 1.000 & 0.750 & 1.250 \\
\hline & Caretta caretta & 1.000 & 0.750 & 1.250 \\
\hline & Lepidochelys olivacea & 0.667 & 1.200 & 1.373 \\
\hline \multirow{21}{*}{$\begin{array}{c}\text { Other } \\
\text { species }\end{array}$} & Lepidocybium flavobrunneum & 0.833 & 1.000 & 1.302 \\
\hline & Lampris guttatus & 1.333 & 1.000 & 1.667 \\
\hline & Taractichthys steindachneri & 0.600 & 0.750 & 0.960 \\
\hline & Ruvettus pretiosus & 1.000 & 1.500 & 1.803 \\
\hline & Acanthocybium solandri & 0.714 & 1.500 & 1.661 \\
\hline & Alepisaurus ferox & 1.500 & 1.000 & 1.803 \\
\hline & Alepisaurus brevirostris & 1.250 & 0.750 & 1.458 \\
\hline & Coryphaena hippurus & 0.429 & 1.500 & 1.560 \\
\hline & Masturus lanceolatus & 1.000 & 1.500 & 1.803 \\
\hline & Pteroplatytrygon violacea & 1.500 & 1.667 & 2.242 \\
\hline & Lophotus lacepede & 0.500 & 1.333 & 1.424 \\
\hline & Gempylus serpens & 0.667 & 1.000 & 1.202 \\
\hline & Taractes rubescens & 0.800 & 0.750 & 1.097 \\
\hline & Mobula japanica & 1.833 & 1.250 & 2.219 \\
\hline & Mola mola & 1.000 & 1.000 & 1.414 \\
\hline & Sphyraena barracuda & 1.000 & 1.750 & 2.016 \\
\hline & Assurger anzac & 1.333 & 1.000 & 1.667 \\
\hline & Ranzania laevis & 1.000 & 1.250 & 1.601 \\
\hline & Elagatis bipinnulata & 0.750 & 1.500 & 1.677 \\
\hline & Remora remora & 0.600 & 1.500 & 1.616 \\
\hline & Prognichthys gibbifrons & 0.333 & 2.000 & 2.028 \\
\hline
\end{tabular}


wonus pelamis)는 성숙체장보다 어획 평균체장이 더 커서 낮은 위험 도를 나타내었다. Fig. 4(b)에서 대부분의 새치류도 다랑어류와 유사하게 높은 위험도를 나타냈으며, 돛새치 (Istiophorus platypterus)가 새치류중 가장 높은 위험 도를 나타 내었다. 황새치는 성숙체 장과 어획평균체 장이 비슷하기는 하였으나 어획평균체장이 조금 더 작아서 낮은 위험도를 나타넜으며, 흑새치 (Makaira indica)는 자료 의 없어 분석하지 못했 다. Fig. 4(c) 에서 검목상어(Isistius brasiliensis) 를 제외 한 상어류 11 종에 대한 지표별 위험 도평 가에 서 청새리상어, 청상아리, 홍살귀상어, 산호상어
(Carcharhinus amblyrhynchos) 등 총 5종은 높은 위 험도를 나타낸 반면, 나머지 6종은 낮은 위험도 를 나타냈다. Fig. 4(d)에서 기타어류에서는 쥐가 오리(Mobula japanica)와 쐐기개복치(Ranzania laevis)는 낮은 위험도를 나타낸 반면, 이 두 종을 제외한 10 종의 어종은 높은 위험 도를 나타냈다.

생산력 및 민감도에 대한 특성치별 위험도평가 생산력 및 민감도에 대한 특성치별 위험도평 가에서 Fig. 5 와 Table 3 에서 제시된 것과 같이 다랑어류, 새치류 및 바다거북류에 속하는 대부 분의 종들은 낮거나 중간단계의 위험도, 상어류

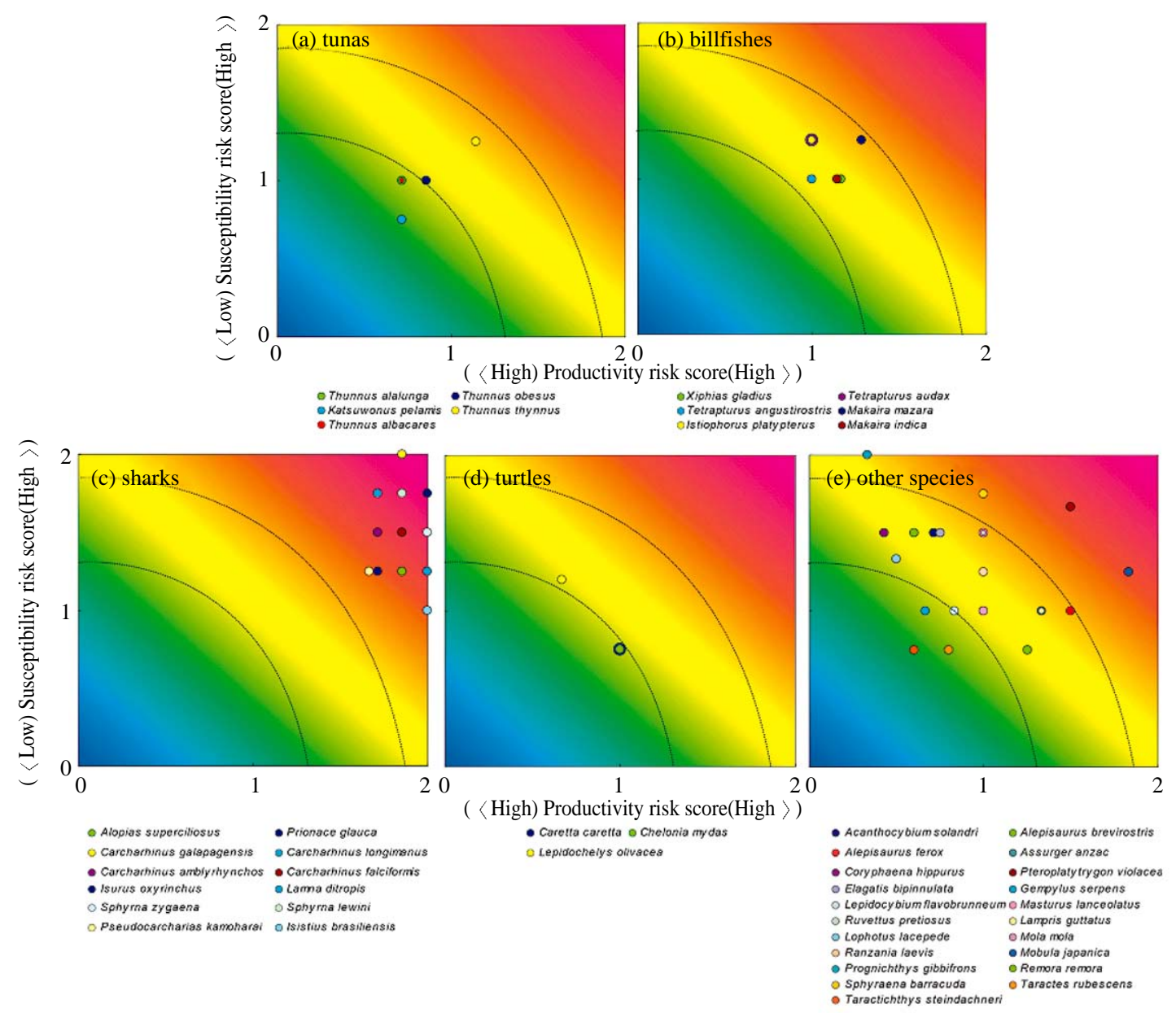

Fig. 5. Plot between productivity and susceptibility analysis of species caught by the Korean tuna longline fisheries during 2005-2008 in the Western and Central Pacific Ocean. 
는 모든 어종이 높은 단계의 위험도, 기타어류는 낮은 단계부터 높은 단계까지의 고른 위험도 경 향을 나타내 는 것으로 평 가되었다.

Fig. 5(a)에서 다랑어류에서는 참다랑어의 위 험도 크기 $(D)$ 가 1.694 로 높은 단계의 위험도에 근접한 중간 단계의 위험도로 평가되었으며, 눈 다랑어 역시가 1.317 로 중간단계의 위험도로 평 가되었다. 날개다랑어 와 황다랑어는 두 어 종 모 두 1.229 로 중간 단계의 위험도에 가까운 낮은 단계의 위험도를 보였으며, 가다랑어는 1.036 으 로 낮은 단계의 위험도로 평 가되었다.

Fig. 5(b)에서 새치류에 속하는 모든 새치들은 중간 단계의 위험도로 평 가되었으며, 그 중 녹새 치 가 높은 단계의 위험단계에 가까운 1.793 으로 중간 단계의 위험도를 나타냈다. 그 외 다른 새 치들은 대부분 비슷한 위험도의 값을 보였으며, 그 중 단문청새치(Tetrapturus angustirostris) 가 중간 단계의 위험도 중 1.414 로 가장 낮은 값을 보었다.

Fig. 5(c)에서 상어류에 속하는 모든 상어의 위 험도는 높은 단계의 위험도로 평 가되었으며, 대 부분의 어종들이 생산력에 관한 위험도가 높게 평 가 되었 다. 갈라 퐈고스상어(Carcharhinus galapagensis)의 위험도가 2.729 로 상어류 중 가 장 높은 단계의 위험도로 평 가되었으며, 다음으 로 청 상아리, 홍살귀상어, 귀상어, 장완흥상어 의 순으로 평가되 었다.

Fig. 5(d)에서 바다거북류는 푸른바다거북과 붉은바다거북(Caretta caretta)은 1.250 의 값을 보여 낮은 단계의 위험도로 평가되었으며, 꼬마 바다거북(Lepidochelys olivacea)은 1.373 으로 중 간 단계의 위험도로 평 가되었다.

Fig. 5(e)에 서 기타어 류에 속하는 어 종들 은 위 험 도가 0.960 에 서 2.242 까지 낮은 단계에서 높은 단계의 위험도까지 고루 분포하었으며, 흰꼬리 타락치(Taractichthys steindachneri) 가 가장 낮은 단계의 위험도로 평 가되었으며, 보라색가오리 (Dasyatis violacea) 가 가장 높은 단계의 위험도
로 평 가되 었다.

\section{고 찰}

본 연구의 과학옵서버 승선조사에서 연승어 업의 주목표종인 다랑어류가 전체 어획량 중 $56.8 \%$ 였으며, 부수어 획되 는 종의 비율-이 $43.2 \%$ 로 부수어획종이 어획량의 절반정 도를 차지하 였다. 그러나 지난 20 년 동안 한국다랑어연승어 업에서 다랑어류의 어획 비율은 평 균 $90 \%$ 로 보 고되고 있으나, 본 연 구의 비율과 비교 해 볼 때, 일 반적인 상업어선에서는 이용가치가 없는 어 종들에 대해서는 계기나 기록누락 등으로 실제 연 승어업에서 부수어획 비율이 과소평 가되고 있다(Moon et al., 2007). 따라서 본 연 구에서는 상업적 어업에는 보고되지 않고 있으나 한국다 랑어연 승어업에 의해 지속적으로 어획되나 자 원 평가가 이루어지고 있지 않은 비목표종에 대 한 생태학적 위험도를 평 가하였고 특히, 본 연구 에 사용된 생산력 - 민감도 분석법은 생태계기 반 어업관리의 한 방법으로 어업의 지속성 유지 를 위해 대상어업이 생태계에 미치는 영향을 평 가하는 적 당한 방법이 다(Stobutzki et al., 2001).

다랑어류에 대한 성숙체 장 및 어획평균체장 에 대한지표별 비교및 특성치별 비교에서 참다 랑어의 생태학적 위험도가 다랑어류 중 가장 높 은 것으로 평 가되었으며, 눈 다랑어는 낮은 단계 의 위험도에 가까운 중간 단계의 위험도로 평 가 되었다. 이것은 생태학적으로 어업의 영향으로 인해 회복할 수 있는 재 생산율이 높은 위험도를 나타내는 어종일수록 높은 어획노력을 투입할 때 생태학적으로 낮은 위험도를 나타내는 어종 에 비해 자원감소가 더 빨리 일 어나는 것으로 추 정할 수 있다. 정 량적 자원평 가에 서도 역 시 참다 랑어가 눈다랑어나 다른 다랑어류에 비해 자원 이 가장 낮은 어종으로 평가되었으며(FAO, 2007c), 특히, 참다랑어는 1960 년 대 이후 어획노 력 과 자원량은 반비례 관계 를 보었다(Ichinokawa et al., 2007). 참다랑어의 어획량은 지 속적으로 감소하헜고, 최근 참다랑어 어 획량은 1960 년 대 
최고 어획량에 비해 약 2 배정도 감소하였다. 눈 다랑어의 어획량은 1960 년 대 어획량에 비해 최 근에는 약 3 배 이상의 어획량을 보이고 있다 (FAO, 2007b).

가다랑어(Katsuwonus pelamis)는 지표와 특성 치별 비교에서 모두 낮은 단계의 위험도를 나타 냈다. 이는 참다랑어(Thunnus thynnus)나 눈다랑 어(Thunnus obesus)에 비해 가다랑어 가 성숙연 령이 낮고 성숙체 장이 작아 재 생산율이 더 높기 때 문인 것으로 평 가되 었다. 반면에 가다랑어의 생산력에 대한 위험 도는 황다랑어(Thunnus albacares) 와 날개다랑어(Thunnus alalunga) 와 같 은 값으로 평 가되 었으나, 어 구 영향력 에 대 한 민 감도 부분에서는 아주 낮은 위험도로 평 가되었 다. 이는 가다랑어가 주로 표층에서 서식하여 중 층을 주 어획 수심(25-309m)으로 하는 다랑어 연 승어업에서는 많이 어획되지 않았기 때문이 다(FAO, 1993). 눈다랑어, 황다랑어, 날개다랑어 의 경우 현재 중서부태평양해 역의 눈 다랑어 자 원 의 상태는 과도 어획된 상태는 아니지 만자원 이 계속 감소하는 경항을 보이고 있으므로자원 보존을 위해서는 어획노력 및 어획량을 감소를 권 고하는 상태이 다(Langley et al., 2008; Langley et al., 2007; Hoyle et al., 2008). 그러나 가다랑어 의 자원상태는 과도어획의 상태나 징후가 없으 며 자원보존을 위한어뗘한 조치를 취하고 있지 는 않다(Langley and Hampton, 2008). 다랑어 류 에 대한 정 량적 자원평 가와 생태학적 위험도평 가를 비교하였을 때 생태학적 위험도가 높은 어 종일수록 지속적으로 어업에 부정적인 영향을 받게 될 경우 자원감소의 위험이 더 커진다고 할 수 있겠다.

새치류의 지표와 특성에 대한 각각의 비교에 서 황새치(Xiphias gladius) 가 지표별 비교에 서 낮은 위험도를 나타냈으며, 특성별 비교 역시 중 간 단계의 위험도로 평 가되 었다. 이러한 결 과는 황새치자원량이 최근에 증가하는 경향을 나타 내고 있으며, 과도어획의 상태를 보이고 있지 않
은 것으로 평 가한 최 근 황새치 의 정 량적 평 가 결 과를 잘 반영하고 있다(Kolody et al., 2008). 그러 나 녹새치(Makaira mazara)의 경 우 중간 단계의 위험도와 근접한 높은 단계의 위험도로 평가되 었으며, 이는 정 량적 평 가에 서도 자원이 최대 지 속적 생산량시의 자원량 $\left(\mathrm{B}_{\mathrm{MSY}}\right)$ 보다 낮게 추정되 어 자원관리의 필요성이 대두되고 있다(Kleiber et al., 2003).

상어류에 대한 지표별 비교평가에서는 11 종 중 청새리상어(Prionace glauca), 청상아리(Isurus oxyrinchus), 홍살귀 상어(Sphyrna lewini), 강 남 상어(Pseudocarcharias kamoharai), 산호상어 (Carcharhinus amblyrhynchos) 등 5종을 제외한 나머지 6 종은 어 획평 균체 장이 성 숙체 장보다 작 아 낮은 위험도로 평 가되었다. 그러나 생산력 과 민감도에 대한 특성별 비교평 가특히, 한국다랑 어 연 승어업에 대한 영향과 그에 따른 어종의 재 생 산율에 대한 위험도평 가에서는 모든 상어가 높은 단계의 위험도로 평 가되었다. 모든 상어류 는 난태생 어류로 성숙연 령이 평균 10 세 이상으 로 재생산율이 가장 낮은 수준의 어류로 분석되 고, 서식수심이나 분포 범위가 대부분 연 승어구 의 주 어획 수심에 분포하고 있어 민감도에서도 높은 단계의 위험도로 평 가되었다. 이러한 연구 결과는 다랑어 연승어업이 상어류 자원상태에 직 접적인 영향을 미치고 있다는 사실을 잘 반영 하고 있다.

우리나라 다랑어 어획량의 $90 \%$ 이상을 차지 하고 전 세계 최대 다랑어 어장인 중서부태평양 수산위 원회(Western and Central Pacific Fisheries Commission, WCPFC)에서는 2008 년에 상어류 자원 평가 및 관리를 위해 다랑어 연승어업에서 가장 많이 어획 되고, 옵서버나 어업 자에 의해 식 별이 용이 한 청상아리, 장완 흥상어(Carcharhinus longimanus), 큰눈 환도 상어(Alopias superciliosus) 및 청새리상어, 4 종에 대한 각 회원 국별 어입자 료 수집을 권고하였다(WCPFC, 2008). 그러나 위 의 4 종중생 태학적 위험도평가 결 과 큰눈환도상 
어와 청새리상어는 상대적 으로 낮게 평 가된 반 면, 청상아리와 장완흥상어 에 대한 위험도가 높 기는 하나, 갈라파고스상어(Carcharhinus galapagensis), 홍살귀 상어, 귀 상어(Sphyrna zygaena) 에 대한 위험도보다는 낮은 것으로 평가되었다. 생태 학적 위험 도평 가가 어업 의 영향에 대한 어 종의 생물학적 재생산율에 대한 분석이라는 점 을 고려할 때, 상어류 자원 보존을 위해서는 우점 종이나 식별이 용이한 점 외에 좀 더 과학적인 근 거를 기반으로 시급히 자원보존이 취해져야할 종들의 우선순위를 정할 필요가 있다. 따라서 항 후 상어류에 대한 자원 평가 및 관리를 위해서는 $\mathrm{WCPFC}$ 에서 제시한4종보다는 생태 학적 위험도 가 더 높은 갈라퐈고스 상어, 청상아리, 홍살귀상 어, 귀상어에 대한 어업자료가 수집되고 자원 평 가를 통한 관리 방안을 수립 할 필요가 있다.

본 연구는 단일 종에 대한 정량적 평가를 벗어 나 다종자원 평가 및 생태계기 반 자원 평가의 개 념으로 향후 자원 평가 및 관리가 필요한 어종의 우선순위를 결정하는데 도움이 되는 예방적 접 근법으로 사용 가능하며, 생태계의 효율적인 보 존과 관리를 통한 생태계의 종 다양성을 유지와 어업 활동으로 인한 관련 어종 및 생태계의 위험 도를 최소화 할 수 있는 방법으로 활웅 가능 하다.

\section{결 론}

본 연 구에서는 중서부태평양해역의 한국다랑 어연승어업에 의해 영향을 받는 목표종 뿐만아 니라 부수어획되는 모든 종에 대한 생태학적 위 험 도평 가를 실시하였다. 이를 위해 2005 - 2008 년간 중서부태평양 한국 다랑어 연 승어업에 대 한 총 7회에 걸친 과학자 승선조사 자료가 사용 되었다. 생태학적 위험도평가 결 과 다랑어류, 새 치류 및 바다거북류에 속하는 대부분 의 종들이 중간 단계의 위험도로 평 가되었으나, 상어류는 모든 종이 높은 단계의 위험도로 평 가되 었다. 생 산력 - 민감도 분석법 은 한국다랑어 연 승어업에 대한 영향과 그에 따른 어종의 재생산율에 대한
위험도를 평가하는 것이기 때 문에 연구 결 과 다 랑어 연승어업이 상어류 자원상태에 직접적인 영향을 미치고 있다는 사실을 알 수 있다. 중서 부태평양수산위 원회 에서 상어류 자원보존을 위 해서는 우점종이나 식별이 용이한 점 때문에 청 상아리, 장완흥상어, 큰눈환도상어 및 청새리상 어 등 4 종에 대한 자료수집을 권 고하고 있으나, 좀 더 과학적인 근거를 기반으로 시급히 자원보 존이 취해져야할 종들의 우선순위를 정할 필요 가 있다.

\section{사 사}

본 연 구는 국립수산과학원 원 양어 업자원조사 사업의 일환으로 수행되 었으며(RP - 2009 FR - 003), 본 논문 수집에 협조해준 국제옵서버 및 조사선 선장 및 선원 여러분께 감사드 립니다.

\section{참고문헌}

FAO(Food and Agriculture Organization of the United Nations), 2007a. Report of the expert consultation on international guidelines for the management of deep-sea fisheries in the high seas, pp. 48.

FAO, 2007b. Capture production 2005. FAO Yearbook of Fishery Statistics Vol.100/1, pp. 539.

FAO, 2007c. Methodological Workshop on the Management of Tuna Fishing Capacity. Stock status, data envelopment analysis, industry surveys and management options. FAO Fisheries Proceedings P8, pp. 218.

FAO, 1993. Interactions of Pacific tuna fisheries. Volume 2 Papers on biology and fisheries. FAO Fisheries Technical Paper 336/2. pp. 439.

Gilman, E., S. Clarke, N. Brothers, J. Alfaro-Shigueto, J. Mandelman, J. Mangel, S. Petersen, S. Piovano, N. Thomson, P. Dalzell, M. Donson, M. Goren and T. Werner, 2008. Shark interactions in pelagic longline fisheries. Mar. Pol, 32, 1 - 18.

Hoyle1, S., A. Langley, and J. Hampton, 2008. Stock assessment of albacore tuna in the South Pacific Ocean. WCPFC-SC4-2008/SA-WP-8. 4th Regular 
Session of the WCPFC Scientific Committee, Port Moresby, pp. 126.

Ichinokawa, M., M. Kai, Y. Takeuchi and R. Conser, 2007. Brief review of the methods for the future projections of Pacific bluefin tuna stock assessment. ISC/07/PBFWG-3/21, pp. 29.

Kirby, D.S., 2006. An ecological risk assessment for species caught in WCPO longline and purse seine fisheries. Working Paper EB-WP1, 2nd Regular Session of the WCPFC Scientific Committee, Manila, pp. 25.

Kirby, D.S., B. Moloney, 2007. An ecological risk assessment(ERA) for the effects of fishing in the Western \& Central Pacific Ocean: research planning workshop report and draft research plan. Working Paper EB-WP3, 3rd Regular Session of the WCPFC Scientific Committee, Honolulu, pp. 31.

Kleiber, P., M.G. Hinton and Y. Uozumi, 2003. Stock assessment of blue marlin (Makaira nigricans) in the Pacific using MULTIFAN-CL. Marine and Freshwater Research. 54(4), 349 - 360.

Kolody, D., R. Campbell and N. Davies, 2008. A MULTIFAN-CL stock assessment of South-West Pacific swordfish 1952-2007. WCPFC-SC4-2008 /SA-WP-6. 4th Regular Session of the WCPFC Scientific Committee, Port Moresby, pp. 90.

Langley, A. and J. Hampton, 2008. Stock assessment of skipjack tuna in the western and Central Pacific Ocean. WCPFC-SC4-2008/SA-WP-4. 4th Regular Session of the WCPFC Scientific Committee, Port Moresby, pp. 74.

Langley, A., J. Hampton, K. Pierre and S. Hoyle, 2008. Stock assessment of bigeye tuna in the Western and Central Pacific Ocean, including an anlaysis of management options. WCPFC-SC4-2008/SA-WP-1. 4th Regular Session of the WCPFC Scientific Committee, Port Moresby, pp. 137.

Langley, A., J. Hampton, P. Kleiber and S. Hoyle, 2007. Stock assessment of yellowfin tuna in the western and central Pacific Ocean, including an analysis of management options. WCPFC-SC3-SA SWG/WP01. 3rd Regular Session of the WCPFC Scientific Committee, Honolulu, pp. 120.

Moon, D.Y., S.J. Hwang, D.H. An and S.S. Kim, 2007. Bycatch of sharks in Korean tuna longline fishery. J. Kor. Soc. Fish. Tech., 43(4), 392 - 338.

Pikkitch, E.K., C. Santora, E.A. Babcock, A. Bakun, R. Bonfil, D.O. Conover, P. Dayton, 2004. Ecosystem based fishery management. Science, 305, 346 - 347.

Smith, A.D.M., E.J. Fulton, A.J. Hobday, D.C. Smith and P. Shoulder, 2007. Scientific tools to support the practical implementation of ecosystem-based fisheries management. ICES J. Mar. Sci., 64, 633 639.

Stobutzki, I., M. Miller and D. Brewer, 2001. Sustainability of fishery bycatch: a process for assessing highly diverse and numerous bycatch. Environmental Conservation, 28(2), 167 - 181.

WCPFC(Western and Central Pacific Fisheries Commission), 2008. Executive summary. Commission for the Conservation and Management of highly migratory fish stocks in the Western and Central Pacific Ocean. Scientific committee fourth regular session. 11 -22, Aug., 2008, pp. 266.

Yokota, K., M. Kiyota and H. Minami, 2006. Shark catch in a pelagic longline fishery: Comparison of circle and tuna hooks. Fish. Res., 81, 337 - 341.

\footnotetext{
2009년 2월 5일 접수

2009년 2 월 12 일 1 차 수정

2009년 2 월 12 일 수리
} 